\title{
Singularity-free cosmological solutions in quadratic gravity
}

\author{
P. Kanti, J. Rizos and K. Tamvakis \\ Division of Theoretical Physics, Physics Department, \\ University of Ioannina, Ioannina GR 451 10, Greece.
}

\begin{abstract}
We study a general field theory of a scalar field coupled to gravity through a quadratic GaussBonnet term $\xi(\phi) R_{G B}^{2}$. The coupling function has the form $\xi(\phi)=\phi^{n}$, where $n$ is a positive integer. In the absence of the Gauss-Bonnet term, the cosmological solutions for an empty universe and a universe dominated by the energy-momentum tensor of a scalar field are always characterized by the occurrence of a true cosmological singularity. By employing analytical and numerical methods, we show that, in the presence of the quadratic Gauss-Bonnet term, for the dual case of even $n$, the set of solutions of the classical equations of motion in a curved FRW background includes singularity-free cosmological solutions. The singular solutions are shown to be confined in a part of the phase space of the theory allowing the non-singular solutions to fill the rest of the space. We conjecture that the same theory with a general coupling function that satisfies certain criteria may lead to non-singular cosmological solutions.
\end{abstract}

PACS numbers: $98.80 . \mathrm{Hw}, 04.50 .+\mathrm{h}, 11.25 . \mathrm{Mj}, 04.20 . \mathrm{Jb}$

Emails : pkanti@cc.uoi.gr, irizos@cc.uoi.gr, tamvakis@cc.uoi.gr 


\section{INTRODUCTION}

Despite the successes of Einstein's theory of Gravitation at large distances, a Quantum Theory of Gravity, valid at supersmall distances, requires a more general framework. At present, Superstring Theory [1] seems to provide the most appealing framework for such a theory. Superstring Theory leads to the unification of gravity with the other fundamental forces. It also leads to important modifications of the standard cosmology, based on the Einstein action, at short distances of the order of the Planck length. Although the theory has not been fully developed to the point that a detailed cosmology could be constructed, a number of general conclusions can be drawn regarding new possibilities that distinguish string cosmology from the standard model. Modifications of gravity of stringy origin can be studied through the Superstring Effective Action corrected by incorporating loop and $\alpha^{\prime}$ effects. The latter are associated with the contribution of the infinite tower of massive string modes, while the former are due to quantum loop effects. Although the full String Theory is approximated only in a perturbative sense by the Effective Action and this is expected to describe Physics only up to energies where quantum gravitational effects start becoming dominant, it is hoped that the loop-corrected Action captures many of the true features of the exact theory. The study of the loop-corrected Superstring Action has uncovered interesting possibilities [2] not realized by the Einstein-Hilbert action such as the existence of novel stable dilatonic black holes [3] that circumvent the "no hair" theorem in its restricted sense. There are of course alternative approaches to string cosmology. The absence of cosmological singularities in the presence of higher-curvature terms, in various numbers of spacetime dimensions, has been pointed out in the literature several times [4]. Another approach is the pre-Big Bang scenario [5] which attempts to incorporate features of the exact theory such as duality symmetries.

A remarkable property of the loop-corrected Superstring Effective Action in the presence of the dilaton and moduli fields is the existence of singularity-free solutions with flat initial asymptotics [6]. These are linked to the $R^{2}$ gravitational terms with field-dependent coefficients that are present. These solutions which avoid the initial singularity are possible for a definite sign of the corresponding trace anomaly for which the strong energy conditions related to the modulus energy-momentum tensor can be violated. They start from a flat space-time in the infinite past, they pass through an inflationary period and they end up as a slowly expanding universe. A general field theory of a scalar field coupled to gravity through a quadratic Gauss-Bonnet term $\xi(\phi) R_{G B}^{2}$ has also been shown to possess singularity-free solutions in a spatially flat FRW background under very mild assumptions on the coupling function $\xi(\phi)$ [7]. In a subsequent paper by Easther and Maeda [ [B], the case of a closed FRW universe based on the loop-corrected Superstring Action was also shown to lead, via numerical methods, to such singularity-free solutions.

In the article at hand, being inspired by the Superstring Effective Theory, we consider a generic theory with a scalar field coupled to gravitation through the higher-curvature quadratic Gauss-Bonnet term. For simplicity, we keep only the one-loop gravitational quantities that appear in the action functional of the Superstring Effective Theory. For the case 
of a spherically symmetric background that we are going to consider, the other one-loop gravitational quantity $R \tilde{R} \equiv \eta^{\mu \nu \kappa \lambda} R_{\mu \nu}^{\sigma \tau} R_{\kappa \lambda \sigma \tau}$ vanishes identically. This model captures the essential features and was shown to possess non-singular solutions in the flat case for a general class of coupling functions [7]. Here, we extend the analysis of Ref. [7] to the case of a curved universe, both open or closed. We develop a purely analytical argument and we manage to show that the singular cosmological solutions, characterized by a true singularity at finite time, are indeed present in the theory but they are confined in a certain part of the phase space of the theory. In this way, the non-singular cosmological solutions are summoned to fill the rest of the space. These results are radically different from those that follow from the same theory when the quadratic Gauss-Bonnet term is absent. In that case, as we will show, the singular cosmological solutions cover the whole phase space of the theory leaving no room for the existence of non-singular solutions.

The structure of this article is as follows: In section 1, we derive the equations of motion for the scalar and gravitational fields in a curved FRW background. In section 2, we study the cosmological solutions of the theory when the Gauss-Bonnet term is absent. We consider both the cases of an empty universe and a universe dominated by the energy-momentum tensor of a scalar field. In section 3, we develop our analytical argument for the existence of non-singular cosmological solutions in the presence of the Gauss-Bonnet term. In section 4, a numerical analysis for a specific choice of the coupling function serves as an illuminating example for our theory. The last, short section is devoted to our conclusions.

\section{Equations of motion of the theory}

We consider the quadratic coupling of a scalar field with gravity through the GaussBonnet term which is described by the action

$$
S=\int d^{4} x \sqrt{-g}\left\{\frac{R}{2}+\frac{1}{2} \partial_{\mu} \phi \partial^{\mu} \phi-\frac{1}{16} \delta \xi(\phi) \mathcal{R}_{G B}^{2}\right\}
$$

Note that $\xi(\phi)$ is, for the time being, a general coupling function. The Gauss-Bonnet term is defined as

$$
\mathcal{R}_{G B}^{2}=R_{\mu \nu \rho \sigma} R^{\mu \nu \rho \sigma}-4 R_{\mu \nu} R^{\mu \nu}+R^{2}
$$

and $\delta$, which in Superstring Effective Theory is proportional to the trace anomaly of the theory, plays the role of a coupling parameter.

The spacetime background assumes the standard spherical symmetric FRW form

$$
d s^{2}=d t^{2}-e^{2 \omega(t)}\left\{\frac{d r^{2}}{1-k r^{2}}+r^{2}\left(d \theta^{2}+\sin ^{2} \theta d \varphi^{2}\right)\right\}
$$

where $k=0, \pm 1$ corresponding to the flat, closed and open universe, respectively. 
Making use of the above metric components and assuming further that the scalar field $\phi$ depends solely on the time coordinate $t$, the equations of motion take the form

$$
\begin{aligned}
& \ddot{\phi}+3 \dot{\phi} \dot{\omega}-24 \frac{d f}{d \phi}\left(\ddot{\omega}+\dot{\omega}^{2}\right)\left(\dot{\omega}^{2}+k e^{-2 \omega}\right)=0, \\
& 2\left(\ddot{\omega}+\dot{\omega}^{2}\right)(1+8 \dot{f} \dot{\omega})+\left(\dot{\omega}^{2}+k e^{-2 \omega}\right)(1+8 \ddot{f})+\frac{1}{2} \dot{\phi}^{2}=0, \\
& 3\left(\dot{\omega}^{2}+k e^{-2 \omega}\right)(1+8 \dot{f} \dot{\omega})-\frac{\dot{\phi}^{2}}{2}=0,
\end{aligned}
$$

where $f(\phi)=-\delta \xi(\phi) / 16$. If we set $x=\dot{\phi}, z=\dot{\omega}$ and $y=e^{-2 \omega}$, we obtain

$$
\begin{aligned}
& \dot{x}+3 z x+\frac{3}{2} \delta \xi^{\prime}\left(\dot{z}+z^{2}\right)\left(z^{2}+k y\right)=0 \\
& \left(\dot{z}+z^{2}\right)\left(4-2 \delta \xi^{\prime} x z\right)+\left(z^{2}+k y\right)\left(2-\delta \xi^{\prime \prime} x^{2}-\delta \xi^{\prime} \dot{x}\right)+x^{2}=0 \\
& \left(z^{2}+k y\right)\left(6-3 \delta \xi^{\prime} x z\right)-x^{2}=0
\end{aligned}
$$

Rearranging eqs. (1.7) and (1.8), we obtain a new equation which contains only the time derivative of $z$

$$
\dot{z}=\frac{d z}{d \phi} x=-z^{2}-\frac{\left(2-\delta \xi^{\prime \prime} x^{2}+3 \delta \xi^{\prime} z x\right)\left(z^{2}+k y\right)+x^{2}}{4-2 \delta \xi^{\prime} z x+\frac{3}{2}\left(\delta \xi^{\prime}\right)^{2}\left(z^{2}+k y\right)^{2}}
$$

while, from the definition of $y$ we are led to the following differential equation

$$
\dot{y}=\frac{d y}{d \phi} x=-2 y z .
$$

On the other hand, we may solve eq. (1.9) as an algebraic equation and write $x$, the time derivative of the scalar field $\phi$, as a function of $z, y$ and $\delta \xi^{\prime}$ in the following way

$$
x=-\frac{3}{2} \delta \xi^{\prime} z\left(z^{2}+k y\right)+s \sqrt{\left[\frac{3}{2} \delta \xi^{\prime} z\left(z^{2}+k y\right)\right]^{2}+6\left(z^{2}+k y\right)} \quad, \quad s= \pm 1
$$

Note that the set of equations (1.10)-(1.11) is characterized by an invariance under the simultaneous change of the signs of $z$ and $s$. In order to clarify this point, we suppose that we have found a solution, for the choice $s=+1$, described by the set of equations

$$
\begin{aligned}
& \dot{z}=\frac{d z}{d \phi} x_{+}=-z^{2}-\frac{\left(2-\delta \xi^{\prime \prime} x_{+}^{2}+3 \delta \xi^{\prime} z x_{+}\right)\left(z^{2}+k y\right)+x_{+}^{2}}{4-2 \delta \xi^{\prime} z x_{+}+\frac{3}{2}\left(\delta \xi^{\prime}\right)^{2}\left(z^{2}+k y\right)^{2}}, \\
& \dot{y}=\frac{d y}{d \phi} x_{+}=-2 y z .
\end{aligned}
$$


where $x_{+}$stands for the value of $x$ that corresponds to the choice $s=+1$. Under the transformation $z \rightarrow-z$, the set of equations (1.13)-(1.14) is replaced by a new one with $x_{-}$in the place of $x_{+}$, where $x_{-}$corresponds to the choice $s=-1$. This means that if $x_{+}$ corresponds to a solution (singular or not) of the equations of motion, then $x_{-}$corresponds to the same solution with the sign of $z$ reversed. For this reason, we may keep fixed the sign of $z$, e.g. $z>0$, during the analytical treatment of the problem.

\section{The $\delta=0$ case}

We first consider the case with $\delta=0$, that is without the Gauss-Bonnet term. If we further assume that the scalar field takes on a constant value and set $x \equiv \dot{\phi}=0$ in equations (1.4)-(1.6), we obtain

$$
\begin{aligned}
& \dot{\omega}^{2}+k e^{-2 \omega}=0, \\
& \ddot{\omega}+\dot{\omega}^{2}=0 .
\end{aligned}
$$

For $k=0$, we obtain $\omega=$ const. which corresponds to a static universe with arbitrary radius. On the other hand, for $k=+1$, we must have $\omega=$ const. and $e^{-\omega}=0$ at the same time, which corresponds to a static universe with infinite radius. The only interesting case is the last one, $k=-1$, where we find that $e^{\omega(t)}=a(t) \sim t$. This result corresponds to a linearly, eternally expanding universe with an initial singularity, at $t=0$. Note that the rate of expansion is much larger than in the case of the "radiation" $\left(a \sim t^{1 / 2}\right)$ or the "matter" $\left(a \sim t^{2 / 3}\right)$ epoch of the Standard Cosmological Model. This is due to the absence of any matter content of the universe capable of slowing down the expansion of the universe.

Next, we allow the scalar field to evolve with time $(\dot{\phi} \neq 0)$ while keeping the parameter $\delta$ equal to zero. In this case, the set of equations (1.10)-(1.11) take the form

$$
\begin{aligned}
& \dot{z}=\frac{d z}{d \phi} x=-3 z^{2}-2 k y, \\
& \dot{y}=\frac{d y}{d \phi} x=-2 y z
\end{aligned}
$$

where

$$
x=s \sqrt{6\left(z^{2}+k y\right)} \quad, \quad s= \pm 1 .
$$

We are going to study separately the cases of flat and curved space :

A) Flat Space $(k=0)$ : The solution of (2.3)-(2.4) with respect to time $t$ takes the form

$$
\dot{z}=-3 z^{2} \Rightarrow z(t)=(c+3 t)^{-1}
$$




$$
\begin{aligned}
& \dot{y}=-2 y z \Rightarrow y(t)=(c+3 t)^{-2 / 3}, \\
& \dot{\phi}=s \sqrt{6} z \Rightarrow \phi(t)=s \sqrt{\frac{2}{3}} \ln (c+3 t)+c^{\prime}
\end{aligned}
$$

where $c$ and $c^{\prime}$ are arbitrary constants. The result for the scale factor of the universe is

$$
e^{\omega}=a(t)=(c+3 t)^{1 / 3}
$$

which corresponds to an expanding universe with a true cosmological singularity at finite time. Note that, here, the rate of expansion of the universe is smaller than the corresponding ones during the two epochs of the Standard Cosmological Model. The sole reason for this result is the presence of the energy momentum tensor of the free scalar field $\phi$ on the righthand side of the Einstein's equations which leads to the slowing down of the expansion of the universe in a more effective way than the energy momentum tensor of a perfect fluid.

B) Curved Space $(k= \pm 1)$ : In this case, the system (2.3) may be reduced to a single equation :

$$
\dot{z}=\frac{d z}{d y} \dot{y}=\frac{d z}{d y}(-2 y z)=-3 z^{2}-2 k y \Rightarrow 2 y d z-3 z d y=\frac{2 k y}{z} d y
$$

If we multiply both sides by $z / y^{4}$, we obtain

$$
d\left(\frac{z^{2}}{y^{3}}\right)=(-k) d\left(\frac{1}{y^{2}}\right) \Rightarrow z^{2}+k y=c_{1} y^{3}
$$

where $c_{1}$ is a positive constant. Substituting the above in the differential equation of $y$, we get the result

$$
\begin{gathered}
\dot{y}=\frac{d y}{d \phi} x=\frac{d y}{d \phi} s \sqrt{6 c_{1} y^{3}}=-2 y \sqrt{y\left(c_{1} y^{2}-k\right)} \Rightarrow \\
\Rightarrow y=\frac{c_{1} k+c_{2}^{2} \exp \left\{-2 s \sqrt{\frac{2}{3}} \phi\right\}}{2 c_{1} c_{2} \exp \left\{-s \sqrt{\frac{2}{3}} \phi\right\}} .
\end{gathered}
$$

From the above expression as well as from eq. (2.9), it is evident that there is a further invariance of the solutions under the interchange of the signs of $s$ and $\phi$. As a result, we may keep fixed the sign of $s$, e.g. $s=+1$, while allowing $\phi$ to take on both positive and negative values.

A cosmological singularity is encountered when $a(t) \rightarrow 0$ or equivalently when $y \rightarrow \infty$. From the expression (2.10), we conclude that, when $k=+1, y$ goes to infinity for $\phi \rightarrow \pm \infty$ while, for $k=-1$, a singular behaviour arises only for $\phi \rightarrow-\infty$. Near the singularities, we 
may evaluate the approximate expression of $y$ which can be written in the following way

$$
\begin{array}{ll}
\text { For } \quad \phi \rightarrow-\infty \Rightarrow y \simeq \frac{c_{2}}{2 c_{1}} e^{-\sqrt{\frac{2}{3}} \phi} & (\text { for } k= \pm 1), \\
\text { For } \quad \phi \rightarrow+\infty \Rightarrow y \simeq \frac{k}{2 c_{2}} e^{\sqrt{\frac{2}{3}} \phi} \quad(\text { for } k=+1) .
\end{array}
$$

The corresponding expressions for $z$ can be easily derived from eq. (2.8) and exist only when the solution for $y$ exists as well. By making use of the differential equation for $\phi$, eq. (2.4), we may deduce the dependence of the scalar field on time $t$ near the singularities and, consequently, the expression of the scale factor of the universe in the same region. Then, we obtain

$$
\begin{array}{ll}
a(t) \simeq\left(c^{\prime}+3 \sqrt{c_{1}} t\right)^{1 / 3} & (\text { for } k= \pm 1) \\
a(t) \simeq\left(c^{\prime}-3 \sqrt{c_{1}} t\right)^{1 / 3} & (\text { for } k=+1) .
\end{array}
$$

The above expressions describe also a universe with a true cosmological singularity at finite time. We note that for $k=+1$, that is for the case of a closed universe, there are always two branches of singular solutions with vanishing $a(t)$. On the other hand, for the options $k=0,-1$ which correspond to the cases of a flat and open universe, respectively, there is only one branch of singular solutions. This result is in perfect agreement with the singularity content of the Standard Cosmological Model. The open and flat universe are characterized by only one singularity, the initial one, while in the case of a closed universe we encounter two cosmological singularities, the initial and the final one.

It is also worth noting that, for the choice $\delta=0$, the group of singular solutions found above covers the whole phase space of the theory leaving no space for the existence of nonsingular solutions. The final singularity of the closed universe (2.14) can be avoided only if we choose $k=0,-1$. On the other hand, the initial singularity (2.13) disappears only if we set $c_{2}=0$. Then, we end up with the totally unrealistic case of a static universe with infinite radius. As a result, we conclude that, in the absence of the Gauss-Bonnet term, the only realistic cosmological solutions, that we may obtain in the framework of the theory (1.1), contain, at least, one true singularity.

\section{The $\delta \neq 0$ case}

In this section, we are going to search for non-singular cosmological solutions in the presence of the quadratic Gauss-Bonnet term in the action functional of the theory. It will be useful for our analysis to search for violations of the energy conditions [9] that indicate absence of singularities. Assuming a perfect fluid form for the energy-momentum tensor of the system, 
the energy and pressure are defined as : $T_{00}=\rho, T_{i i}=-p g_{i i}$. Using the equations of motion (1.4)-(1.6), the energy conditions take the form

$$
\begin{aligned}
& \rho+p=-2\left(\ddot{\omega}-k e^{-2 \omega}\right)=2\left(k y+z^{2}\right) \frac{B+24 x^{2} z^{4}-6 \delta \xi^{\prime \prime} x^{4} z^{2}\left(z^{2}+k y\right)}{B}, \\
& \rho+3 p=-6\left(\dot{\omega}^{2}+\ddot{\omega}\right)=\frac{36 x^{2} z^{2}}{B}\left(8-\delta \xi^{\prime \prime} x^{2}\right)\left(k y+z^{2}\right)^{2},
\end{aligned}
$$

where

$$
\begin{aligned}
B= & x^{4} k y+5 x^{4} z^{2}-12 x^{2} k^{2} y^{2}-24 x^{2} k y z^{2}-12 x^{2} z^{4}+ \\
& 108 k y z^{4}+36 k^{3} y^{3}+108 k^{2} y^{2} z^{2}+36 z^{6}
\end{aligned}
$$

and where we have used eq. (1.5) in order to eliminate $\delta \xi^{\prime}$. In this form, we may easily prove that for $k=0,+1$ the term $B$, being a polynomial with respect to $x^{2}$ with no real roots, is always positive definite. Thus the energy conditions can only be violated, leading to non-singular cosmological solutions, for $\delta>0$. For $k=-1$, the analysis is much more complicated but it can be shown that the energy conditions are violated for both signs of $\delta$. However, by making use of numerical as well as analytical arguments, we may show that non-singular solutions arise only for $\delta>0$, too. As a result, in our analysis, we may consider $\delta$ to be always positive.

Next, we will try to determine all the singular solutions of the theory with the singularity occurring at finite time hoping that they do not cover the whole phase space leaving some room for the non-singular ones. The whole treatment will be analytical assuming a polynomial dependence of the coupling function $\xi(\phi)$ on the scalar field, $\xi(\phi)=\phi^{n}$ with $n$ being a positive integer greater than unit. Since a singular solution is characterized by the vanishing of the scale factor at some finite time, $a(t) \equiv e^{\omega(t)} \rightarrow 0$, we will always demand that near the singularity $y \equiv e^{-2 \omega} \rightarrow \infty$. In the same region, the quantity $z \equiv \dot{\omega}$ will be set to approach a constant value, zero or infinity while the scalar field $\phi$ will be left free to adopt any possible behaviour.

We are going to concentrate our attention on the study of the following cases :

$\underline{\text { (I) } z=\text { finite } \neq 0, \phi=\text { any, } y \rightarrow \infty .}$

If, near the singularity, $z$ remains finite adopting a constant value, $z(t)=c$, then, from the differential equation for $y$ (1.11), we obtain

$$
\frac{\dot{y}}{y}=-2 z \Rightarrow y=e^{-2 \omega} \sim e^{-2 \int z(t) d t} \Rightarrow R(t) \sim e^{c t}
$$

which goes to zero only when $c t \rightarrow-\infty$. This means that the singularity is approached only at infinite time and for this reason it must be excluded. 
In this case, the first derivative of the scalar field with respect to time takes the form

$$
x=-\frac{3}{2} \delta \xi^{\prime} z k y+s \sqrt{\left(\frac{3}{2} \delta \xi^{\prime} z k y\right)^{2}+6 k y} .
$$

We have to consider the following cases:

(A) $\left(\frac{3}{2} \delta \xi^{\prime} z k y\right)^{2} \gg 6 k y \Rightarrow \frac{3}{8}\left(\delta \xi^{\prime} z\right)^{2} k y \gg \mathcal{O}(1)$. Then, $x$ can be written as

$$
\begin{aligned}
x & \simeq-\frac{3}{2} \delta \xi^{\prime} z k y+\frac{3 s}{2} z y \delta\left|\xi^{\prime} k\right|+\frac{2 s k}{z \delta\left|\xi^{\prime} k\right|}= \\
& =\left\{\begin{array}{ccc}
\text { if } \quad s=+1 \quad \Rightarrow \quad x_{+}=\frac{2}{\delta \xi^{\prime} z}, \\
\text { if } \quad s=-1 \quad \Rightarrow \quad x_{-}=-3 \delta \xi^{\prime} z k y .
\end{array}\right.
\end{aligned}
$$

The above values of $x$ have been taken for the case $\xi^{\prime} k>0$. If we change the sign of $\xi^{\prime}$ according to $\xi^{\prime} \rightarrow-\xi^{\prime}$, we obtain $x_{ \pm} \rightarrow-x_{\mp}$. On the other hand, if we change the sign of $k$ in the same way, we are led to $x_{ \pm} \rightarrow \mp x_{\mp}$. Note that, apart from the interchange $x_{+} \leftrightarrow x_{-}$, the only thing that changes is the absolute sign of $x$ which is not going to be used in the following analysis. For this reason, we may consider only the cases $\xi^{\prime}>0$ and $k>0$.

We are going to study each expression of $x$ separately:

(a) $x=x_{+}=\frac{2}{\delta \xi^{\prime} z}$. Then, eq. (1.10) reduces to :

$$
\dot{z}=\frac{d z}{d \phi} \frac{2}{\delta \xi^{\prime} z} \simeq-z^{2}\left\{1-\frac{8 \delta \xi^{\prime \prime}}{3\left(\delta \xi^{\prime} z\right)^{4} k y}\right\} .
$$

If $\frac{8 \delta \xi^{\prime \prime}}{3\left(\delta \xi^{\prime} z\right)^{4} k y} \ll \mathcal{O}(1)$, then, rearranging the differential equations for $z$ and $y$, we obtain : $2 y \dot{z}=z \dot{y} \Rightarrow z^{2} \sim y$ which is inconsistent with our assumption for the behaviour of $z$ near the singularity. Next, we assume that $\frac{8 \delta \xi^{\prime \prime}}{3\left(\delta \xi^{\prime} z\right)^{4} k y}=b \simeq \mathcal{O}(1)$. In the same way, we obtain $z^{2} \simeq y^{(1-b)}$ which is consistent with our assumptions only for $(1-b)<0$. However, the differential equation for $y$ gives the result $y^{(b-1)} \sim(t+c)$, which leads to the conclusion that the singularity is approached only at infinite time and for this reason it must be excluded. If, finally, $\frac{8 \delta \xi^{\prime \prime}}{3\left(\delta \xi^{\prime} z\right)^{4} k y} \gg \mathcal{O}(1)$, we are led to

$$
\left.\begin{array}{l}
\dot{z}=\frac{8 \delta \xi^{\prime \prime}}{3\left(\delta \xi^{\prime}\right)^{4} z^{2} k y} \Rightarrow \frac{d\left(z^{2}\right)}{d \phi}=\frac{8}{3} \frac{(\delta \xi)^{\prime \prime}}{\left(\delta \xi^{\prime}\right)^{3} k y} \\
\dot{y}=-2 y z \Rightarrow \frac{d\left(z^{2}\right)}{d \phi}=-\left(\frac{y^{\prime}}{y \delta \xi^{\prime}}\right)^{\prime}
\end{array}\right\} \Rightarrow
$$




$$
\Rightarrow \quad y^{\prime \prime}\left(\delta \xi^{\prime}\right)^{2} y-y^{\prime 2}\left(\delta \xi^{\prime}\right)^{2}-y^{\prime} y \delta \xi^{\prime \prime} \delta \xi^{\prime}+\frac{8}{3 k} \delta \xi^{\prime \prime} y=0
$$

If we assume that $\xi(\phi)=\phi^{n}$, the only solution of the above differential equation, compatible with this assumption, is the following

$$
y=\frac{b_{1}}{\delta \xi^{\prime \prime}} \quad \text { and } \quad y^{\prime}=\frac{b_{2}}{\delta \xi^{\prime}}
$$

provided that

$$
b_{1}=\frac{8(n-1)^{2}}{3 k n(2-n)} \quad \text { and } \quad b_{2}^{2}+2 b_{2} b_{1}-\frac{8}{3} b_{1}=0
$$

But, then, we obtain :

$$
\left(\delta \xi^{\prime} z\right)^{2} y=\left(\delta \xi^{\prime}\right)^{2}\left(-\frac{y^{\prime}}{y \delta \xi^{\prime}}\right) y=-y^{\prime} \delta \xi^{\prime}=-b_{2}=\mathcal{O}(1)
$$

which is inconsistent with our assumption that $\left(\delta \xi^{\prime} z\right)^{2} y \gg \mathcal{O}(1)$.

(b) $x=x_{-}=-3 \delta \xi^{\prime} z k y$. Then, eq. (1.10) takes the form

$$
\dot{z}=\frac{d z}{d \phi}\left(-3 \delta \xi^{\prime} z k y\right) \simeq-z^{2}\left(1-6 \delta \xi^{\prime \prime} k y\right) .
$$

If we assume that $6 \delta \xi^{\prime \prime} k y \ll \mathcal{O}(1)$ or $6 \delta \xi^{\prime \prime} k y=b \simeq \mathcal{O}(1)$, we obtain exactly the same result as in case (a). The first assumption leads to infinite $z$ while the second one leads to a singularity which is approached only at infinite time. The third option, $6 \delta \xi^{\prime \prime} k y \gg \mathcal{O}(1)$, leads to

$$
\dot{z}=\frac{d z}{z \phi}\left(-3 \delta \xi^{\prime} z k y\right) \simeq z^{2} 6 \delta \xi^{\prime \prime} k y \Rightarrow z \simeq\left(\delta \xi^{\prime}\right)^{-2}
$$

which goes to zero only if $\phi \rightarrow \infty$. By using the above result and for $\xi(\phi)=\phi^{n}$ with $n>2$, the differential equation for $y$ gives : $y \sim\left(\delta \xi^{\prime \prime}\right)^{-1}$ or equivalently that $\delta \xi^{\prime \prime} y \simeq \mathcal{O}(1)$ which is inconsistent with our assumption that $\delta \xi^{\prime \prime} y \gg \mathcal{O}(1)$. For the special case of $n=2$, the same equation leads to $y \sim \ln \phi$ and consequently to $\left(\delta \xi^{\prime} z\right)^{2} y \ll \mathcal{O}(1)$ which is again inconsistent with the assumption $\left(\delta \xi^{\prime} z\right)^{2} y \gg \mathcal{O}(1)$.

(B) $\left(\frac{3}{2} \delta \xi^{\prime} z k y\right)^{2} \ll 6 k y \Rightarrow \frac{3}{8}\left(\delta \xi^{\prime} z\right)^{2} k y \ll \mathcal{O}(1)$. Then, $x \simeq \pm \sqrt{6 k y}$, which means that the following analysis is valid only for $k=+1$. The differential equation for $z$ takes the form :

$$
\dot{z}=\frac{d z}{d \phi} x \simeq-z^{2}-\frac{\left(8-6 \delta \xi^{\prime \prime} y\right) y}{4+\frac{3}{2}\left(\delta \xi^{\prime}\right)^{2} y^{2}}
$$

Now, we have to consider the following cases concerning the quantity $\delta \xi^{\prime} y$ that appears in the denominator of the above equation : 
(a) $\delta \xi^{\prime} y=a=\mathcal{O}(1)$. This means that $\phi \rightarrow 0$ and consequently that $\delta \xi^{\prime \prime} y \gg \mathcal{O}(1)$. Then, the differential equation for $z$ takes the form

$$
\dot{z} \simeq \frac{6 \delta \xi^{\prime \prime} y^{2}}{A} \Rightarrow d z \simeq \pm \frac{\sqrt{6}}{A} d\left(\delta \xi^{\prime}\right) y \sqrt{y},
$$

where $A=4+\frac{3}{2} a^{2}$. Since $\delta \xi^{\prime} y=$ const., we easily obtain $d\left(\delta \xi^{\prime}\right) y=-\delta \xi^{\prime} d y$. Then, we find that $z \sim \sqrt{y}$ which once again leads to a behaviour of $z$ radically different from the assumed.

(b) $\delta \xi^{\prime} y \gg \mathcal{O}(1)$. In this case, and according to our assumption that $\xi(\phi)=\phi^{n}$, we can only have : $\delta \xi^{\prime \prime} y \gg \mathcal{O}(1)$. Then, we obtain

$$
\left.\begin{array}{l}
\frac{d z}{d \phi} x \simeq \frac{4 \delta \xi^{\prime \prime}}{\left(\delta \xi^{\prime}\right)^{2}} \\
\frac{d( \pm \sqrt{6 y})}{d \phi}=\frac{d x}{d \phi}=-z
\end{array}\right\} \Rightarrow x^{\prime \prime} x+\frac{4 \delta \xi^{\prime \prime}}{\left(\delta \xi^{\prime}\right)^{2}}=0 .
$$

The only solution of the above differential equation, compatible with our assumption for $\xi(\phi)$, is the following

$$
x=\frac{b_{1}}{\sqrt{\delta \xi^{\prime \prime}}} \quad, \quad \text { where } \quad b_{1}^{2}=\frac{16(n-1)^{2}}{n(2-n)} .
$$

However, the above result leads to $\delta \xi^{\prime \prime} y \sim \mathcal{O}(1)$ which is inconsistent with our assumption that $\delta \xi^{\prime \prime} y \gg \mathcal{O}(1)$. Moreover, the above solution for $x$ is real only if $n<2$ which is in disagreement with our assumption for $\xi(\phi)$.

(c) $\delta \xi^{\prime} y \ll \mathcal{O}(1)$. In this case, the differential equation for $z$ takes the simple form $\dot{z} \simeq$ $-\left(2-\frac{3}{2} \delta \xi^{\prime \prime} y\right) y$. For the assumptions $\delta \xi^{\prime \prime} y \ll \mathcal{O}(1)$ and $\delta \xi^{\prime} y=b \simeq \mathcal{O}(1)$, we obtain the result $z^{2} \sim y$ which is different from our assumption that $z$ goes to zero near the singularity. The other option, $\delta \xi^{\prime \prime} y \gg \mathcal{O}(1)$, is a little more complicated as it leads to

$$
\left.\begin{array}{l}
\frac{d z}{d \phi} x \simeq \frac{3}{2} \delta \xi^{\prime \prime} y^{2} \\
\frac{d( \pm \sqrt{6 y})}{d \phi}=\frac{d x}{d \phi}=-z
\end{array}\right\} \Rightarrow x^{\prime \prime}+\frac{\delta \xi^{\prime \prime} x^{3}}{24}=0 .
$$

In the same way, the only solution of the above differential equation, for $\xi(\phi)=\phi^{n}$, is the following

$$
x=\frac{b_{2}}{\sqrt{\delta \xi}} \quad, \quad \text { where } \quad b_{2}^{2}=\frac{6(n+2)}{(1-n)} .
$$

This means that the above solution is real only for $n<1$ which is inconsistent with our assumption for $\xi(\phi)$. 
C) $\left(\frac{3}{2} \delta \xi^{\prime} z k y\right)^{2} \simeq 6 k y \Rightarrow \frac{3}{8}\left(\delta \xi^{\prime} z\right)^{2} k y=\frac{1}{a} \simeq \mathcal{O}(1)$. Then, $x \simeq \frac{3 \lambda}{2} \delta \xi^{\prime} z k y$, where $\lambda=$ $-1 \pm \sqrt{1+a}$, and eq. (1.10) takes the form :

$$
\dot{z}=-z^{2}\left\{(1+2 a)-\frac{3 \lambda^{2}}{2} \delta \xi^{\prime \prime} k y\right\}
$$

Since $\left(\delta \xi^{\prime} z\right)^{2} y \simeq \mathcal{O}(1)$, we will always have $\delta \xi^{\prime \prime} y \gg \mathcal{O}(1)$ independently of the behaviour of the scalar field $\phi$ near the singularity. By using the above in the differential equation for $z$, we obtain that $z \sim\left(\delta \xi^{\prime}\right)^{\lambda}$ which goes to zero for $(\lambda>0, \phi \rightarrow 0)$ or $(\lambda<0, \phi \rightarrow \infty)$. The solution of the differential equation for $y$ is $y \sim\left(\delta \xi^{\prime \prime}\right)^{-1}+c$ which leads to $y \simeq \mathcal{O}(1)$ if $\phi \rightarrow \infty$ or to $y \delta \xi^{\prime \prime} \simeq \mathcal{O}(1)$ if $\phi \rightarrow 0$. The first of these results is inconsistent with the assumed behaviour of $y$ near the singularity while the second one disagrees with our assumption that $y \delta \xi^{\prime \prime} \gg \mathcal{O}(1)$.

From the study of the first two cases, (I) and (II), we conclude that in a singular cosmological solution the first derivative of the quantity $\omega$ cannot remain finite or vanish near the singularity. The only option left is the adoption of an infinite value, just like $y$, which we are going to study now.

(III) $z \rightarrow \infty, \phi=$ any, $y \rightarrow \infty$.

We are going to study the following cases :

$$
\text { (A) }\left(\frac{3}{2} \delta \xi^{\prime} z\right)^{2}\left(z^{2}+k y\right)^{2} \gg 6\left(z^{2}+k y\right) \Rightarrow\left(\delta \xi^{\prime} z\right)^{2}\left(z^{2}+k y\right) \gg \mathcal{O}(1) \text {. }
$$

As in case (II), the change of sign of $\xi^{\prime}$ or $z^{2}+k y$ leads to the interchange $x_{+} \leftrightarrow x_{-}$as well as to the change of the absolute sign of $x$. However, the sign of $x$ does not appear anywhere in our analysis while the interchange between the two expressions of $x$ does not affect our arguments since both of them are being studied. As a result, we are going to assume again that both of $\xi^{\prime}$ and $z^{2}+k y$ always take on positive values. Then, under the above assumption, the quantity $x$ assumes the expressions $x_{+}=2 /\left(\delta \xi^{\prime} z\right)$ and $x_{-}=-3 \delta \xi z\left(z^{2}+k y\right)$ for $s= \pm 1$, respectively.

(a) $x=x_{+}=\frac{2}{\delta \xi^{\prime} z}$. Then,

$$
\dot{z}=\frac{d z}{d \phi} \frac{2}{\delta \xi^{\prime} z} \simeq-z^{2}\left\{1-\frac{8}{3} \frac{\delta \xi^{\prime \prime}}{\left(\delta \xi^{\prime} z\right)^{4}\left(z^{2}+k y\right)}\right\} .
$$

First, we assume that $\frac{\delta \xi^{\prime \prime}}{\left(\delta \xi^{\prime} z\right)^{4}\left(z^{2}+k y\right)} \ll \mathcal{O}(1)$. Rearranging the system of differential equations for $z$ and $y$, we easily obtain that $z^{2} \sim y$ which means that the two quantities have exactly the same behaviour near the singularity and, if necessary, their sum may be written as $z^{2}+k y=a z^{2}$, where $a$ an arbitrary constant. The differential equation for $z$ gives the result $z^{2}=(\delta \xi+c)^{-1}$ which goes to infinity when $(\delta \xi+c) \rightarrow 0$. However, we have to 
check that this limit takes place at finite time. From the expression of $x$, we obtain

$$
\frac{d \phi}{d t}=\frac{2}{\delta \xi^{\prime}} \sqrt{\delta \xi+c} \Rightarrow(\delta \xi+c) \sim\left(t+c^{\prime}\right)^{2}
$$

which indeed goes to zero at finite time. It is easy to check further that our basic assumptions $\left(\delta \xi^{\prime} z\right)^{2}\left(z^{2}+k y\right) \gg \mathcal{O}(1)$ and $\frac{\delta \xi^{\prime \prime}}{\left(\delta \xi^{\prime} z\right)^{4}\left(z^{2}+k y\right)} \ll \mathcal{O}(1)$ are satisfied provided that $\delta \xi^{\prime} \neq 0$. As a result, we conclude that the solution

$$
z^{2} \sim y \sim \frac{1}{\delta \xi(\phi)-\delta \xi\left(\phi_{s}\right)}
$$

is an acceptable singular cosmological solution with the singularity being approached at finite time.

Next, we consider the case $\frac{\delta \xi^{\prime \prime}}{\left(\delta \xi^{\prime} z\right)^{4}\left(z^{2}+k y\right)}=b \simeq \mathcal{O}(1)$. Since both $z$ and $y$ are infinite near the singularity and in order to satisfy the above constraint, we must have $\delta \xi^{\prime} \rightarrow 0$. The corresponding solution for $z$ is $z^{-2}=(1-b)(\delta \xi+c)$. If $z^{2} \ll y$, we obtain $\left(\delta \xi^{\prime} z\right)^{2}\left(z^{2}+k y\right) \simeq\left(\delta \xi^{\prime} z\right)^{2} y \gg \mathcal{O}(1)$ and that $z^{2} \sim y^{(1-b)}$. Then :

$$
\frac{\delta \xi^{\prime \prime}}{\left(\delta \xi^{\prime} z\right)^{4} y}=\frac{3 b}{8} \Rightarrow\left(\delta \xi^{\prime}\right)^{-2} \sim z^{2} y+c^{\prime}
$$

The above result leads to $\left(\delta \xi^{\prime} z\right)^{2} y \simeq \mathcal{O}(1)$ which is inconsistent with our assumption. Following an exactly similar analysis, we may show that when $z^{2} \gg y$ or $z^{2} \sim y$, the above integration yields a constraint which is again in obvious disagreement with our assumption that $\left(\delta \xi^{\prime} z\right)^{2}\left(z^{2}+k y\right) \gg \mathcal{O}(1)$.

Finally, we assume that $\frac{\delta \xi^{\prime \prime}}{\left(\delta \xi^{\prime} z\right)^{4}\left(z^{2}+k y\right)} \gg \mathcal{O}(1)$. If, furthermore, $z^{2} \sim y$, we may set $z^{2}+k y=a z^{2}$ and the differential equation for $z$ gives $z^{4} \sim\left(\delta \xi^{\prime}\right)^{-2}$. But, this leads to $\left(\delta \xi^{\prime} z\right)^{2}\left(z^{2}+k y\right) \simeq \mathcal{O}(1)$ which is inconsistent with our assumption. If $z^{2} \gg y$, we reach the same result as in the case $z^{2} \sim y$. Finally, the case $z^{2} \ll y$ has been studied in (IIAa) and has been shown to lead to the result $\left(\delta \xi^{\prime} z\right)^{2}\left(z^{2}+k y\right) \simeq \mathcal{O}(1)$ which is inconsistent with our assumption.

(b) $x=x_{-}=-3 \delta \xi^{\prime} z\left(z^{2}+k y\right)$. In this case, the equation for $z$ takes the form

$$
\dot{z} \simeq-z^{2}\left\{1-\frac{6 \delta \xi^{\prime \prime}\left(z^{2}+k y\right)^{2}}{4 z^{2}+\left(z^{2}+k y\right)}\right\} .
$$

We start by assuming that $\frac{6 \delta \xi^{\prime \prime}\left(z^{2}+k y\right)^{2}}{4 z^{2}+\left(z^{2}+k y\right)} \ll \mathcal{O}(1)$. Then, from the differential equations for $z$ and $y$, we obtain $z^{2} \sim y$ which leads to $\delta \xi^{\prime \prime} z^{2} \ll \mathcal{O}(1)$. Setting $z^{2}+k y=a z^{2}$ and integrating the equation for $z$, we find that $\delta \xi^{\prime} z^{2} \sim \phi+\ldots$ from which we conclude that the scalar field must go to infinity near the singularity. Unfortunately, this result leads to $\delta \xi^{\prime \prime} z^{2} \sim \delta \xi^{\prime \prime} \phi\left(\delta \xi^{\prime}\right)^{-1} \simeq \mathcal{O}(1)$ which is inconsistent with $\delta \xi^{\prime \prime} z^{2} \ll \mathcal{O}(1)$. 
If we assume that $\frac{6 \delta \xi^{\prime \prime}\left(z^{2}+k y\right)^{2}}{4 z^{2}+\left(z^{2}+k y\right)}=b \simeq \mathcal{O}(1)$ and, furthermore, that $z^{2} \sim y$, the differential equation for $z$ gives the result : $\delta \xi^{\prime} z^{2} \sim \phi+\ldots$ which goes to infinity only when $\phi \rightarrow \infty$. Substituting this in the same equation, we obtain that $z \sim \phi^{\frac{2 a(1-b)}{(4 a+4 b-3 a b)}}$. In order to assure the assumed behaviour of $z$ near the singularity, we demand the positivity of the exponent of $\phi$. But, then we conclude that $\xi^{\prime}(\phi)<\phi$ which is inconsistent with our assumption that $\xi(\phi)=\phi^{n}$ with $n>1$. For the case $z^{2} \gg y$, we obtain the same result with $a=1$. Finally, if $z^{2} \ll y$, the two constraints become $\delta \xi^{\prime} y \gg \mathcal{O}(1)$ and $6 \delta \xi^{\prime \prime} y=b$. From the differential equation for $y$, we obtain that $y \delta \xi^{\prime} \sim \phi+\ldots$ which again goes to infinity only if $\phi \rightarrow \infty$. Substituting this result in the same equation, we find that $y \sim \phi^{4 /(4+b)}$ with $4+b>0$. But, this leads once again to the inconsistent result $\xi^{\prime}(\phi)<\phi$.

Finally, we consider the case where $\frac{6 \delta \xi^{\prime \prime}\left(z^{2}+k y\right)^{2}}{4 z^{2}+\left(z^{2}+k y\right)} \gg \mathcal{O}(1)$. If $z^{2} \sim y$, the differential equation for $z$ gives $z \sim\left(\delta \xi^{\prime}\right)^{-2 a /(4+a)}$ which leads to $\delta \xi^{\prime} z^{2} \sim\left(\delta \xi^{\prime}\right)^{(4-3 a) /(4+a)}$. If we set, $y=(a-1) z^{2}$, the differential equation for $y$ gives the result $z \sim \phi^{2 a /(3 a-4)}$. If $a>4 / 3$ and $\phi \rightarrow 0$, the constraint $\delta \xi^{\prime} z^{2} \gg \mathcal{O}(1)$ is satisfied but $z \rightarrow 0$. The same holds if $a<4 / 3$ and $\phi \rightarrow \infty$. In both cases, the behaviour of $z$ is inconsistent with the one that has been assumed. If $z^{2} \gg y$, the equation for $z$ gives $z \sim\left(\delta \xi^{\prime}\right)^{-2 / 5}$ which goes to infinity only when $\delta \xi^{\prime} \rightarrow 0$. But then, $\delta \xi^{\prime} z^{2} \sim\left(\delta \xi^{\prime}\right)^{1 / 5} \rightarrow 0$ which does not agree with our assumption. Finally, if $z^{2} \ll y$, we obtain that $z \sim\left(\delta \xi^{\prime}\right)^{-2}$ which goes to infinity only if $\phi \rightarrow 0$. Then, the differential equation for $y$ gives $y \sim\left(\delta \xi^{\prime \prime}\right)^{-1}+c$ or equivalently $y \delta \xi^{\prime \prime} \simeq \mathcal{O}(1)$ which is inconsistent with our assumption.

(B) $\left(\frac{3}{2} \delta \xi^{\prime} z\right)^{2}\left(z^{2}+k y\right)^{2} \gg 6\left(z^{2}+k y\right) \Rightarrow\left(\delta \xi^{\prime} z\right)^{2}\left(z^{2}+k y\right) \ll \mathcal{O}(1)$.

In this case, the expression of $x$ becomes $x= \pm \sqrt{6\left(z^{2}+k y\right)}$ while the differential equation for $z$ takes the form

$$
\dot{z} \simeq-z^{2}\left\{1+\frac{\left[8-6 \delta \xi^{\prime \prime}\left(z^{2}+k y\right)\right]\left(z^{2}+k y\right)}{4 z^{2}+\frac{3}{2}\left(\delta \xi^{\prime} z\right)^{2}\left(z^{2}+k y\right)^{2}}\right\} .
$$

In order to simplify the analysis, we are going to consider first the possible relation between $z^{2}$ and $y$ and then study each case separately.

(a) $z^{2} \sim y$. Then, the constraint becomes $\delta \xi^{\prime} z^{2} \ll \mathcal{O}(1)$ and the differential equation for $z$ reduces to

$$
\dot{z} \simeq-z^{2}\left[(1+2 a)-\frac{3}{2} a^{2} \delta \xi^{\prime \prime} z^{2}\right],
$$

where we have set $z^{2}+k y=a z^{2}$. If we assume that $\delta \xi^{\prime \prime} \ll \mathcal{O}(1)$, the above equation gives the result $z \sim e^{ \pm(1+2 a) \phi / \sqrt{6 a}}$ which goes to infinity only if $\phi \rightarrow \pm \infty$. But, then, $\delta \xi^{\prime} \rightarrow \infty$ and there is no way that the constraint $\delta \xi^{\prime} z^{2} \ll \mathcal{O}(1)$ can be satisfied. A similar result arises when $\frac{3}{2} a^{2} \delta \xi^{\prime \prime} z^{2}=b \simeq \mathcal{O}(1)$. If we assume that $\delta \xi^{\prime \prime} z^{2} \gg \mathcal{O}(1)$, we find that $z^{2} \sim\left(\delta \xi^{\prime}+c\right)^{-1}$ 
which goes to infinity only when $\delta \xi^{\prime}+c \rightarrow 0$. But, then, the quantity $\delta \xi^{\prime} z^{2}$ goes to infinity as well and this is inconsistent with our initial assumption.

(b) $z^{2} \gg y$. In this case, the results are similar to the ones of the previous case and they easily arise from them if we set $a=1$.

(c) $z^{2} \ll y$. In this case, the constraint becomes $\left(\delta \xi^{\prime} z\right)^{2} y \ll \mathcal{O}(1)$ and the differential equation for $z$ takes the form

$$
\dot{z} \simeq-z^{2}-\frac{\left(8-6 \delta \xi^{\prime \prime} k y\right) k y}{4+\frac{3}{2}\left(\delta \xi^{\prime} k y\right)^{2}}
$$

The above equation has been arisen also and studied in case (IIB). There, it was shown that, for the cases $\delta \xi^{\prime} y \gg$ or $\ll \mathcal{O}(1)$, there was no real solution of the corresponding differential equation for $x$ compatible with the assumption that $\xi(\phi)=\phi^{n}$ with $n \geq 2$. On the other hand, for the case $\delta \xi^{\prime} y=a \simeq \mathcal{O}(1)$, we were led to $z \sim \sqrt{y}$ which is again inconsistent with our assumption that $z^{2} \ll y$.

(C) $\left(\frac{3}{2} \delta \xi^{\prime} z\right)^{2}\left(z^{2}+k y\right)^{2} \simeq 6\left(z^{2}+k y\right) \Rightarrow \frac{3}{8}\left(\delta \xi^{\prime} z\right)^{2}\left(z^{2}+k y\right)=\frac{1}{a} \simeq \mathcal{O}(1)$.

Since both of $z$ and $y$ approach infinity near the singularity, the only way to fulfill the above condition is to have $\phi \rightarrow 0$. The expression of $x$ becomes $x=\frac{3}{2} \lambda \delta \xi^{\prime} z\left(z^{2}+k y\right)$, where $\lambda=-1 \pm \sqrt{1+a}$, while the differential equation for $z$ takes the form

$$
\dot{z} \simeq-z^{2}-\frac{\left(8 a-\delta \xi^{\prime \prime} a x^{2}\right)\left(z^{2}+k y\right)}{4 a-8 \lambda+4\left(z^{2}+k y\right) / z^{2}} .
$$

We consider the cases :

(a) $z^{2} \sim y$. Then, we may set $z^{2}+k y=\beta z^{2}=\tilde{\beta} y$ and the equation for $z$ reduces to

$$
\dot{z} \simeq-z^{2}\left\{1+\frac{4 a \beta}{2 a-4 \lambda+2 \beta}-\frac{3 \lambda^{2} \beta^{2} \delta \xi^{\prime \prime} z^{2}}{2 a-4 \lambda+2 \beta}\right\} .
$$

We assume further that $\delta \xi^{\prime \prime} z^{2} \ll \mathcal{O}(1)$ or equivalently that $\delta \xi^{\prime \prime} y \ll \mathcal{O}(1)$. Then, the differential equation for $y$ gives the result $\delta \xi^{\prime} y \sim\left(\phi^{-1}+c\right)$ which leads to $\delta \xi^{\prime} y \simeq \mathcal{O}(1)$ or, since $y \sim z^{2}$, to $\delta \xi^{\prime} z^{2} \simeq \mathcal{O}(1)$. Substituting this in the above equation for $z$, we obtain that $z \sim e^{-B \phi}$, where $B$ is a combination of $a, \lambda$ and $\beta$. This means that near the singularity, $z \rightarrow 0$ which is inconsistent with the assumed behaviour of $z$. If we assume that $\delta \xi^{\prime \prime} z^{2}=b \simeq$ $\mathcal{O}(1)$, we are led to exactly the same result. Finally, we consider the case $\delta \xi^{\prime \prime} z^{2} \gg \mathcal{O}(1)$. The equation for $z$ leads to the result $z \sim\left(\delta \xi^{\prime}\right)^{2 \lambda \beta /(2 a-4 \lambda+2 \beta)}$ and in order to fulfill the condition that $\delta \xi^{\prime} z^{2} \simeq \mathcal{O}(1)$ we demand that $2 a-4 \lambda+2 \beta+4 \lambda \beta=0$. By using the dependence of $\lambda$ on $a$, we find that the solution of the above algebraic equation is : $\beta=1=-a$. This means that $z^{2}+k y=\beta z^{2}=z^{2}$ or equivalently that $z^{2} \gg y$ which is inconsistent with our 
assumption.

(b) $z^{2} \gg y$. Now, the constraint becomes $\left(\delta \xi^{\prime} z^{2}\right)^{2}=\frac{8}{3 a}$ while the differential equation for $z$ is the same as in the previous case where we have set $\beta=1$. The first two options, namely $\delta \xi^{\prime \prime} z^{2} \ll \mathcal{O}(1)$ and $\delta \xi^{\prime \prime} z^{2}=b \simeq \mathcal{O}(1)$, lead to the inconsistent result $z \rightarrow 0$. The third option, $\delta \xi^{\prime \prime} z^{2} \gg \mathcal{O}(1)$, leads, as above, to an algebraic equation with the sole solution $a=-1$. However, from our constraint, it follows that $a$ is always a positive constant which reveals the inconsistency of this solution as well.

(c) $z^{2} \ll y$. Then, the differential equation for $z$ takes the form

$$
\dot{z} \simeq-z^{2}\left\{(1+2 a)-\frac{3 \lambda^{2}}{2} \delta \xi^{\prime \prime} y\right\} .
$$

We start by assuming that $\delta \xi^{\prime \prime} y \ll \mathcal{O}(1)$. In this case, the differential equation for $y$ gives the result $\delta \xi^{\prime} y \sim \phi+c \simeq \mathcal{O}(1)$ since, as we concluded above, $\phi \rightarrow 0$ near the singularity. Then, we obtain that $\left(\delta \xi^{\prime} z\right)^{2} y \sim\left(z^{2} / y\right) \ll \mathcal{O}(1)$ which is clearly in disagreement with our initial assumption. The same result arises for the case $\delta \xi^{\prime \prime} y=b \simeq \mathcal{O}(1)$. Finally, we assume that $\delta \xi^{\prime \prime} y \gg \mathcal{O}(1)$. Then, as in case (IIC), we obtain that $y \sim\left(\delta \xi^{\prime \prime}\right)^{-1}+c$ or equivalently that $\delta \xi^{\prime \prime} y \simeq \mathcal{O}(1)$ which is again inconsistent with our assumption that $\delta \xi^{\prime \prime} y \gg \mathcal{O}(1)$.

So, the only singular solution found, with the singularity occurring at finite time, has the form

$$
z^{2} \sim y \sim \frac{1}{\delta \xi(\phi)-\delta \xi\left(\phi_{s}\right)} \sim \frac{1}{\left(t-t_{s}\right)^{2}},
$$

where $\phi_{s}$ and $t_{s}$ stand for the value of the scalar field and time, respectively, at the singularity. Since $y \equiv e^{-2 \omega}$, the scale factor of the universe in this case behaves near the singularity as

$$
a(t) \sim\left(t-t_{s}\right) \longrightarrow 0 \text { when } t \rightarrow t_{s}
$$

which corresponds to a linearly expanding universe with a true cosmological singularity at $t=t_{s}$. During our analysis, no restriction on the value of the parameter $k$ has been arisen and, for this reason, the above singularity can be considered either as an initial or as a final one. Note, that the rate of expansion is much larger that the corresponding ones during the "radiation" or the "matter" epoch of the Standard Cosmological model. The same result was found in the first section in the case of an empty universe. However, when the presence of the energy momentum tensor of the scalar field was taken into account the linear dependence of the scale factor on time $t$ changed into the milder dependence $\sim t^{1 / 3}$. Now, after the addition of the Gauss-Bonnet term in the theory, we find again a scale factor linearly dependent on time. This could be interpreted in the following way : although the addition of the Gauss-Bonnet term has made the search for singular cosmological solutions much more complicated, the net result of its presence in the theory is to cancel exactly the contribution of the energy momentum tensor of the scalar field leaving behind an "empty", linearly expanding universe. The only difference between these two cases is that while the truly empty universe gives rise only to an open universe, as one would expect, the virtually "empty" Gauss-Bonnet universe can be interpreted as a closed or open universe. 
The question which arises next is whether the presence of the Gauss-Bonnet term affects the singular cosmological solutions in such a way that they are restricted in only a region of the phase space of the theory leaving some room for the non-singular ones. The singular

solutions (3.32) were determined under the assumptions $x=\frac{2}{\delta \xi^{\prime} z}$ and $\delta \xi^{\prime} \neq 0$. Then, $x$ tends to zero only asymptotically, when the singularity is approached, keeping a definite sign otherwise. If we assume that the singular solutions are characterized by $x>0$, then we must always have $\xi^{\prime}>0$ since $\delta$ is always positive. If $\xi(\phi)=\phi^{n}$ with $n$ even, the first derivative $\xi^{\prime}$, and hence $x$, is positive only if $\phi$ is positive as well. This leads to the restriction of the singular solutions in the positive $\phi$ half-plane in a $z-\phi$ graph leaving the other half for the non-singular ones. The above argument leads to the selection of even values of $n$ since for odd values the first derivative of the coupling function retains always a positive sign, for all values of $\phi$, and this leads to the non-confinement of the singular solutions in a part of the phase space of the theory.

Finally, it is worth noting that for the cases $k=0$ and $k=-1$ the singular solutions are restricted not only in the positive $\phi$ half-plane for $x>0$ and $\delta>0$ but, moreover, in the positive $z$ quarter-plane. This can be justified if we examine eq. (1.9) written in the form

$$
\left(z^{2}+k y\right)\left(6-3 \delta \xi^{\prime} z x\right)=x^{2} .
$$

If we assume that $k=+1$, then, the value $z=0$ is indeed acceptable by the above equation which means that, in this case, the singular solutions can traverse the axis of $\phi$ at the point where $x^{2}=6 y$ and extend at both the upper and lower $z$ quarter-planes. On the other hand, if $k=-1$, the value $z=0$ must be excluded in order to ensure the reality of $x$. This means that, now, the singular solutions remain confined in one of the two $z$ quarter-planes according to the sign of $z$ that has been initially chosen. Finally, if $k=0$, setting $z=0$ in the above equation leads to the result that the singular solutions traverse the $\phi$-axis at the point where $x=0$. However, this point is only reached near the singularity where $z \rightarrow \infty$. As a result, the value $z=0$ is not an acceptable one for the option $k=0$ and the singular solutions are again restricted in one of the two $z$ quarter-planes.

\section{Numerical Analysis}

In this section, we are going to demonstrate numerically the existence of non-singular solutions in the presence of the Gauss-Bonnet term in the action functional of the theory. The analytical study of the previous section has led to the result that the singular solutions, characterized by the occurring of a cosmological singularity at finite time, exist in the context of the theory but they do not cover the whole phase space. As we will see, it is the nonsingular solutions of the theory that are summoned to fill the rest of the space.

As we have already mentioned, the coupling function of the scalar field to the quadratic Gauss-Bonnet term is of the form $\xi(\phi)=\phi^{n}$ with $n$ even. As an illuminating example, we are 
going to assume the simpler case, that is $n=2$, in our numerical analysis. The system of the differential equations (1.10)-(1.11) together with the algebraic equation (1.12) is numerically integrated yielding a solution for $z \equiv \dot{\omega}$ and $a=e^{\omega}$. Since the case $k=0$ has already been studied in Ref. [7], we are going to concentrate our attention on the other two cases, namely $k= \pm 1$.

The solution for the case $k=+1$ is depicted in Figures 1 and 2 for a family of singular and non-singular solutions. As we have analytically proven in the previous section, the singular solutions, in a $z-\phi$ graph, are confined in the positive $\phi$ half-plane, with the $z$-axis playing the role of a barricade for them, since we have chosen $\delta=0.5>0$. Changing gradually the boundary conditions of the numerical integration, the singular solutions cease to exist as we approach the $z$-axis while the non-singular solutions start to develop and cover the negative $\phi$ half-plane. According to our analysis of section 3, the singular solutions are characterized by the simultaneous divergence of the quantity $z$ and of the quantity $y$ or, equivalently, the vanishing of the scale factor $a$. As we also noted, both of the singular and non-singular solutions can traverse the $\phi$ axis and extend at the upper as well as at the lower $z$ quarter-plane. The value $z=0$, for $k=+1$, is an acceptable value for both families since they obey the same equation (3.34). The dependence of the scale factor of the universe is displayed in Figure 2 where the avoidance of the initial as well as of the final singularity of the closed universe is obvious. Both of Figures 1 and 2 have been drawn for the choice $s=+1$. According to our argument of section 2, the solutions, singular or not, of the equations (1.10)-(1.11) are invariant under the simultaneous change of $z$ and $s$. This means that if we choose $s=-1$, the Figures 1 and 2 will remain unchanged apart from the sign of $z$ in Figure 1 .

The asymptotic form of the non-singular solutions, for the case $k=+1$, for early and late times can be found by making use of the ansatz

$$
\phi=\phi_{0}+\phi_{1} t^{\beta} \quad, \quad \omega=\omega_{0}+\omega_{1} t^{a} .
$$

Substituting the above expressions in the system of equations (1.4)-(1.6), we find that the only acceptable non-singular solution has the form

$$
\phi=\phi_{0}+\frac{2 t}{\sqrt{\delta}} \quad, \quad \omega=\frac{1}{2} \ln \left(\frac{3 \delta}{2}\right)+\frac{\omega_{1}}{\sqrt{|t|}},
$$

where $\phi_{0}$ and $\omega_{1}$ are arbitrary constants. According to the above solution, the asymptotic regions $\phi \rightarrow \pm \infty$ correspond exactly to $t \rightarrow \pm \infty$ modulo a constant coefficient. Then, as it is obvious from Fig. 2 as well, the non-singular solutions are characterized in both limits by the same constant $\omega$ parametrized in terms of the parameter $\delta$, or equivalently by the same constant scale factor $a$, which means that our solutions interpolate between the same static Einstein universe without undergoing a cosmological singularity, either initial of final.

A family of non-singular solutions, for the choice $k=-1$, is depicted in Figures 3 and 4. As we note in Figure 3, the singularity-free solutions cover the whole positive $z$ half-plane without being able to cross the $\phi$-axis since this would lead to an imaginary value of $x$ 
according to eq. (3.34). The singular solutions, although not shown in Figure 3, cover the lower-right-hand-side quarter-plane of the graph, for the choice $\delta=0.5>0$, and they are completely separated from the non-singular ones. In Figure 4, the dependence of the scale factor $a$ on the scalar field is displayed with the absence of the initial singularity of the open universe being obvious. The Figures 3 and 4 have been drawn for the choice $s=-1$. As in the previous case, the alternative choice $s=+1$ leads to the same graphs apart from the sign of $z$ due to the corresponding invariance of the equations (1.10)-(1.11). According to Fig. 4, the scale factor adopts a non-vanishing, constant value in the limit $\phi \rightarrow \infty$ while it increases rapidly as $\phi \rightarrow-\infty$. Given that the dependence of the scalar field $\phi$ on time $t$ is a smooth function leading to the asymptotic behaviour $\phi \rightarrow \pm \infty$ when $t \rightarrow \mp \infty$, we may conjecture that the non-singular open universe interpolates between a static Einstein universe at early times and an expanding universe at late times.

In order to complete the picture, we display the graph of the gravitational scalar curvature $R=g^{\mu \nu} R_{\mu \nu}=6 \ddot{\omega}+12 \dot{\omega}^{2}+6 k e^{-2 \omega}$ versus the scalar field $\phi$, for the cases of the closed and open universe, in Figs. 5 and 6, respectively. The absence of divergences, which implies the absence of cosmological singularities, is obvious in both figures. For the case of the closed universe, $k=+1$, the scalar curvature interpolates between two constant values, as $\phi \rightarrow \pm \infty$, while in the case of the open universe, $k=-1$, the scalar curvature adopts a constant value, in the limit $\phi \rightarrow \infty$, reaches a maximum value near $\phi=0$ and vanishes quickly as we approach the asymptotic region $\phi \rightarrow-\infty$.

\section{Conclusions}

In this article, we have studied a general field theory that describes the coupling of a scalar field to higher-curvature gravity through the quadratic Gauss-Bonnet term. As we mentioned in the Introduction, the presence of this term to the action functional of the Superstring Effective Theory has led to the existence of new black hole solutions and non-singular cosmological solutions. For reasons that will be clarified shortly, we have chosen to study a slightly different quadratic gravitational theory where the coupling function between the scalar field and the Gauss-Bonnet term has the polynomial form $\xi(\phi)=\phi^{n}$, with $n$ being a positive integer.

The classical, scalar and gravitational, equations of motion were solved initially in the absence of the Gauss-Bonnet term. Assuming a constant or a time-evolving scalar field, we determined the cosmological solutions that correspond to an empty universe or to a universe that is dominated by the energy-momentum tensor of a scalar field, respectively. In both cases, the cosmological solutions were found to be characterized by, at least, one true cosmological singularity which could not be avoided if a realistic solution was demanded.

In the presence of the Gauss-Bonnet term, the energy conditions were shown to be violated allowing the existence of non-singular cosmological solutions. For $k=0,+1$ this 
violation holds only for $\delta>0$ while for $k=-1$ the conditions are violated for both signs of $\delta$. However, only for $\delta>0$ non-singular solutions arise in the context of the theory. Next, the equations of motion were solved analytically near the region of the cosmological singularity. A family of singular cosmological solutions was determined and was shown to correspond to a linearly expanding universe, $a(t) \sim\left(t-t_{s}\right)$, with a true cosmological singularity at finite time $t_{s}$. This singularity can be considered as an initial or a final one and the corresponding universe can be interpreted as an open or closed. One of the basic conclusions drawn from this analysis was that these solutions are confined in a part of the phase space of the theory leaving the rest of the space for the non-singular ones. This remarkable feature holds only when the coupling function has the dual, polynomial form $\xi(\phi)=\phi^{n}$, where $\mathrm{n}$ is an even, integer number.

The specific choice $\xi(\phi)=\phi^{2}$ was studied numerically and the solution for the parameter $z$ and the scale factor $a$ was determined. For both values of the parameter $k$, the singular cosmological solutions were confined in a part of the phase space of the theory, as predicted, and completely separated from the non-singular ones. For the case $k=+1$, was even possible to found the asymptotic, analytic form of the non-singular solutions in the limit $t \rightarrow \pm \infty$. According to this form and our numerical results, the universe, for the case $k=+1$, interpolates between the same asymptotic, static Einstein universe with constant scale factor passing through an intermediate phase of expansion-contraction. By making use of the asymptotic form of the non-singular solutions (4.2) for early and late times, we may easily find that the scale factor asymptotically adopts the value $: a(t) \equiv e^{\omega} \simeq \sqrt{\frac{3 \delta}{2}}$. This means that the coupling parameter $\delta$ plays the role of the asymptotic value of the scale factor of the universe which explains the appearance of non-singular cosmological solutions only for positive values of $\delta$. According to the above, in the limit $\delta \rightarrow 0$, that is when we eliminate the Gauss-Bonnet term from the action functional of the theory, the asymptotic value of the scale factor goes to zero and we recover the singular cosmological solutions of section 2. For the case $k=-1$, we may conjecture that the universe interpolates between a static Einstein universe at early times and an expanding universe at late times. For both values of $k$, the absence of cosmological singularities, a feature that owes its existence to the presence of the higher-derivative Gauss-Bonnet term, is obvious.

As is well known, similar results arise in the context of the Superstring Effective Theory where the coupling function between the scalar field and the Gauss-Bonnet term has the form

$$
\xi(\phi)=\ln \left[2 e^{\phi} \eta^{4}\left(i e^{\phi}\right)\right],
$$

where $\eta$ is the Dedekind function. However, the above form and the polynomial form that we have chosen during this article share a number of important characteristics. More analytically : they are both invariant under the change $\phi \rightarrow-\phi$, they both have a global minimum at $\phi=0$ and they both take on an infinite value as $\phi \rightarrow \pm \infty$. As a result, we may state the following "theorem" : Any theory of the form (1.1) that describes the coupling of a scalar field with the Gauss-Bonnet term through a coupling function which (i) is dual, (ii) has a global minimum and (iii) asymptotically tends to infinity may lead to singularity- 
free cosmological solutions. Superstring Effective Theory is a characteristic example of such a theory but it is not the only one. Another choice, as we have demonstrated both analytically and numerically, is the theory (1.1) with $\xi(\phi)=\phi^{2 n}$ and a number of alternative choices may follow as long as the corresponding coupling functions satisfy the three aforementioned criteria.

An important subject which has not been addressed in this article is the stability of our singularity-free solutions. Here, we have focused our attention to the existence itself of these solutions. The question of their stability under linear or even non-linear perturbations is an independent subject which still remains open. If our singularity-free solutions turn out to be stable, that will constitute a great achievement towards the resolution of the initial singularity problem. If, on the other hand, these solutions turn out to be unstable, their collapse in the early universe may have led to the creation of primordial black holes [10]. We hope to come back to these open questions in a future publication.

\section{Acknowledgements}

P. K. would like to acknowledge financial support from the research program ПENE $\Delta-95$ of the Greek Ministry of Science and Technology.

\section{References}

[1] For a review see : M. Green, J. Schwarz and E. Witten, Superstring Theory (Cambridge University Press), Cambridge, 1987.

[2] K.C.K. Chan and R.B. Mann, Class. Quant. Grav. 12 (1995) 1609 ;

E. Elizalde, P. Fosalba-Vela, S. Naftulin and S.D. Oditsov, Phys. Lett. B 352 (1995) 235 ;

J. Koga and K. Maeda, Phys. Rev. D 52 (1995) 7066 ;

G. Veneziano, Helv. Phys. Acta 69 (1996) 553 ;

J.D. Barrow and K.E. Kunze, Phys. Rev. D 55 (1997) 623 ; Phys. Rev. D 56 (1997)

741 ; Phys. Rev. D 57 (1998) 2255 ;

J.D. Barrow and M.P. Dabrowski, Phys. Rev. D 55 (1997) 630 ; Phys. Rev. D 57 (1998)

7204; gr-qc/9803048;

N. Kaloper, Phys. Rev. D 55 (1997) 3394 ;

S. Mukherji, Mod. Phys. Lett. A 12 (1997) 639 ;

R. Poppe and S. Schwager, Phys. Lett. B 393 (1997) 51 ;

S. Kar, Phys. Lett. B 401 (1997) 234 ; 
N. Kaloper and K.A. Olive, Phys. Rev. D 57 (1998) 811 ;

N. Kaloper, I.I. Kogan and K.A. Olive, Phys. Rev. D 57 (1998) 7340 ;

S. Foffa and M. Maggiore, gr-qc/9712006;

A.A. Saharian, hep-th/9712091;

S. Forste, hep-th/9802197;

K. Benakli, hep-th/9804096.

[3] P. Kanti, J. Rizos, N.E. Mavromatos, K. Tamvakis and E. Winstanley, Phys. Rev. D 54 (1996) 5049 ; Phys. Rev. D 57 (1998) 6255 ;

P. Kanti and K. Tamvakis, Phys. Lett. B 392 (1997) 30 ;

T. Torii. H. Yajima and K. Maeda, Phys. Rev. D 55 (1997) 739.

[4] A.A. Starobinsky, Phys. Lett. B 91 (1987) 41 ;

R.H. Brandenberger and C. Vafa, Nucl. Phys. B 316 (1989) 391 ;

A.A. Tseytlin and C. Vafa, Nucl. Phys. B 372 (1992) 443 ;

V. Mukhanov and R. Brandenberger, Phys. Rev. Lett. 68 (1992) 1969 ;

R. Brandenberger, V. Mukhanov and A. Sornborger, Phys. Rev. D 48 (1993) 1629 ;

J.D. Barrow, Phys. Rev. D 48 (1993) 3592 ;

T. Damour and A.M. Polyakov, Nucl. Phys. B 423 (1994) 532 ;

C. Angelantonj, L. Amendola, M. Litterio and F. Occhionero, Phys. Rev. D 51 (1995) 1607 ;

N. Kaloper, R. Madden and K.A. Olive, Nucl. Phys. B 452 (1995) 677 ; Phys. Lett. B 371 (1996) 34 ;

M. Gasperini and G. Veneziano, Phys. Lett. B 387 (1996) 715 ;

S.-J. Rey, Phys. Rev. Lett. 77 (1996) 1929 ; Nucl. Phys. Proc. Suppl. A 52 (1997) 344 ;

R. Easther, K. Maeda and D. Wands, Phys. Rev. D 53 (1996) 4247 ;

S. Bose and S. Kar, Phys. Rev. D 56 (1997) 4444 ;

S. Kalyana Rama, Phys. Rev. Lett. 78 (1997) 1620 ; Phys. Rev. D 56 (1997) 6230 ; Phys. Lett. B 408 (1997) 91;

R. Brustein and R. Madden, Phys. Lett. B 410 (1997) 110 ; Phys. Rev. D 57 (1998) 712 ;

A.A. Al-Nowaiser, M. Ozer and M.O. Taha, gr-qc/9803067.

[5] M. Gasperini and G. Veneziano, Astropart. Phys. 1 (1993) 317 ; Mod. Phys. Lett. A 8 (1993) 3701 ; Phys. Rev. D 50 (1994) 2519 ;

R. Brustein and G. Veneziano, Phys. Lett. B 329 (1994) 429 ;

N. Kaloper, R. Madden and K.A. Olive, Phys. Lett. B371 (1996) 34 ;

M. Gasperini, J. Maharana and G. Veneziano, Nucl. Phys. B 472 (1996) 349 ;

M. Gasperini, M. Maggiore and G. Veneziano, Nucl. Phys. B 494 (1997) 315 ; 
E.J. Copeland, J.E. Lidsey and D. Wands, Nucl. Phys. B 506 (1997) 407 ;

Z. Lalak and R. Poppe, Nucl. Phys. B 507 (1997) 419 ;

M. Maggiore, gr-qc/9709004.

[6] I. Antoniadis, J. Rizos and K. Tamvakis, Nucl. Phys. B 415 (1994) 497.

[7] J. Rizos and K. Tamvakis, Phys. Lett. B 326 (1994) 57.

[8] R. Easther and K. Maeda, Phys. Rev. D 54 (1996) 7252.

[9] R. Penrose, Phys. Rev. Lett. 14 (1965) 57 ;

S. Hawking, Proc. R. Soc. London A 300 (1967) 182 ;

S. Hawking and R. Penrose, Proc. R. Soc. London A 314 (1970) 529.

[10] S. Kawai, M. Sakagami and J. Soda, gr-qc/9802033. 


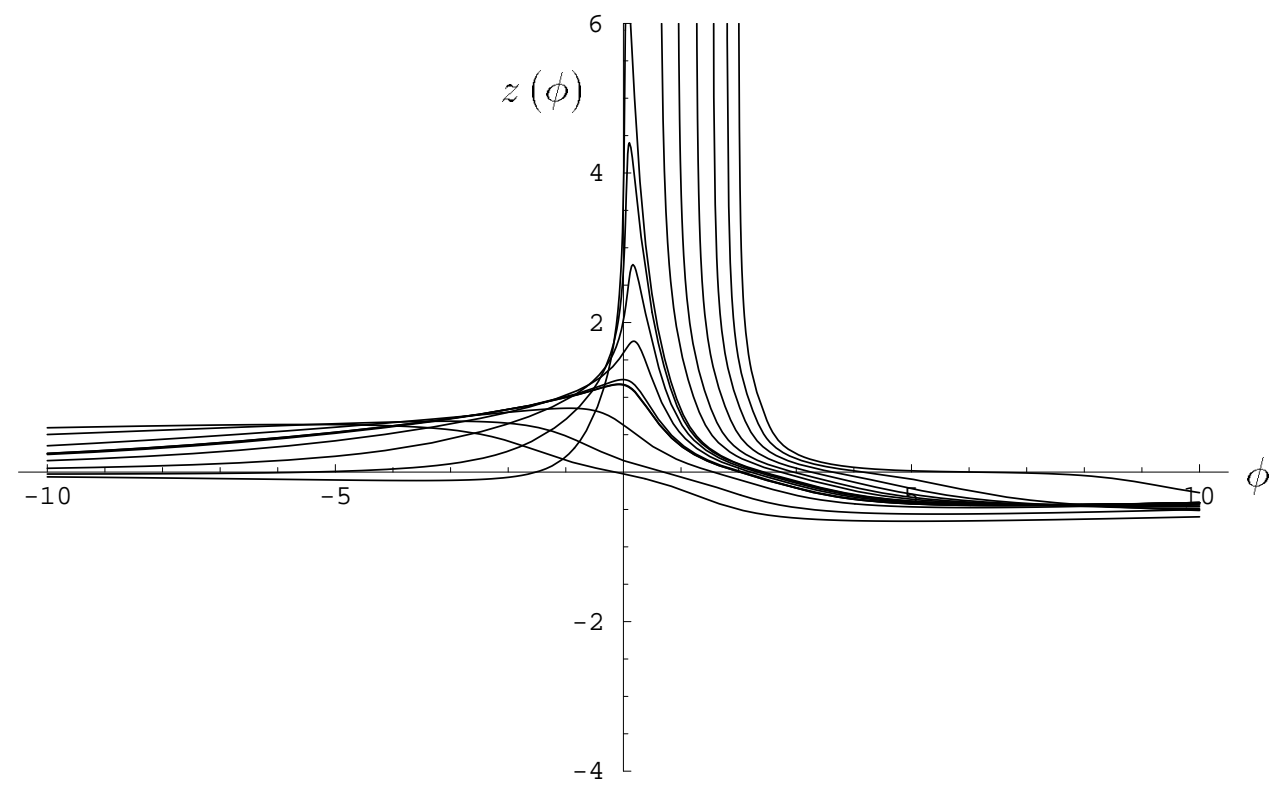

Figure 1: The dependence of the quantity $z$ on the scalar field $\phi$ for a family of singular and non-singular solutions for the case $k=+1, s=+1$ and $\delta=0.5$.

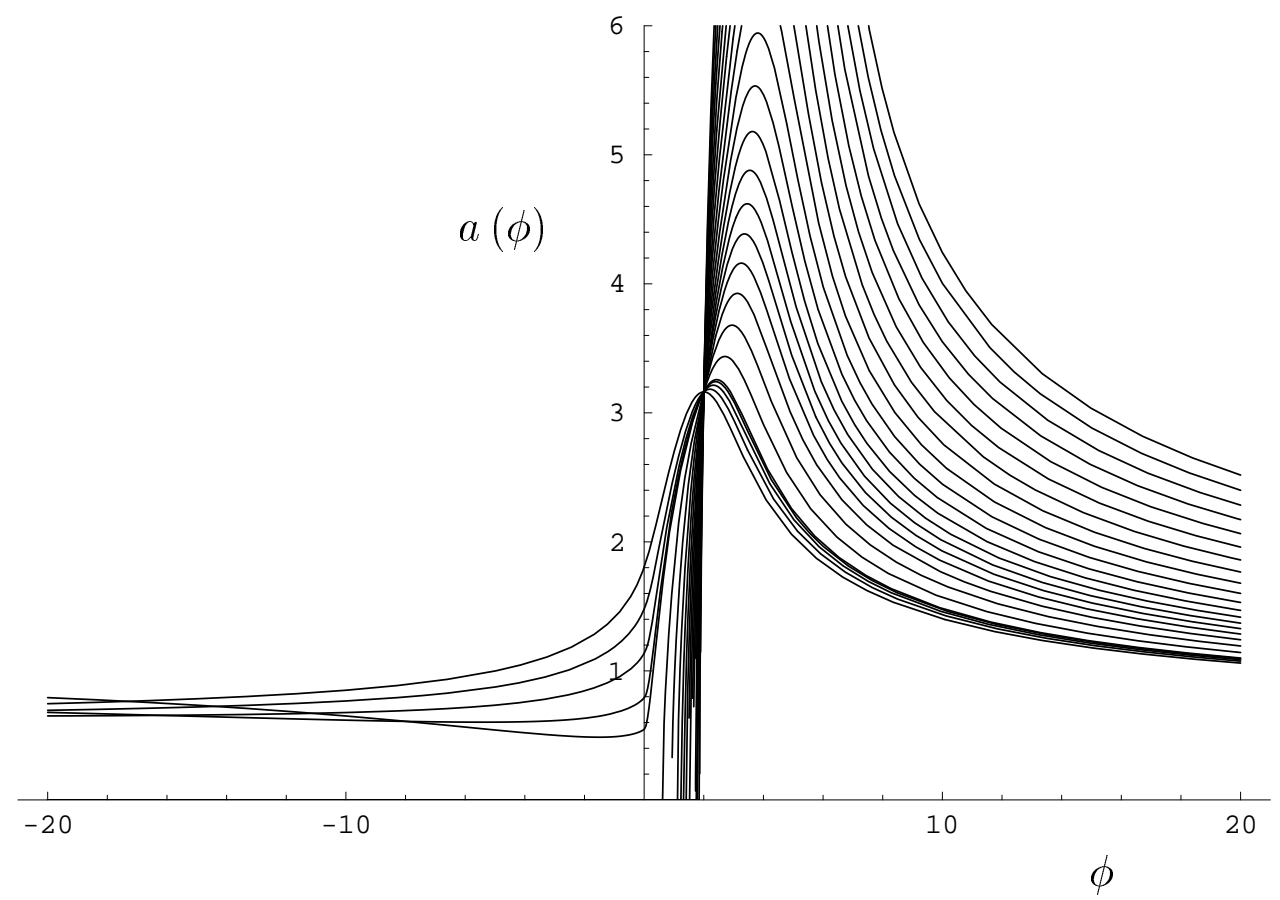

Figure 2: The dependence of the scale factor $a$ of the universe on the scalar field $\phi$ for a family of singular and non-singular solutions for the case $k=+1, s=+1$ and $\delta=0.5$. 


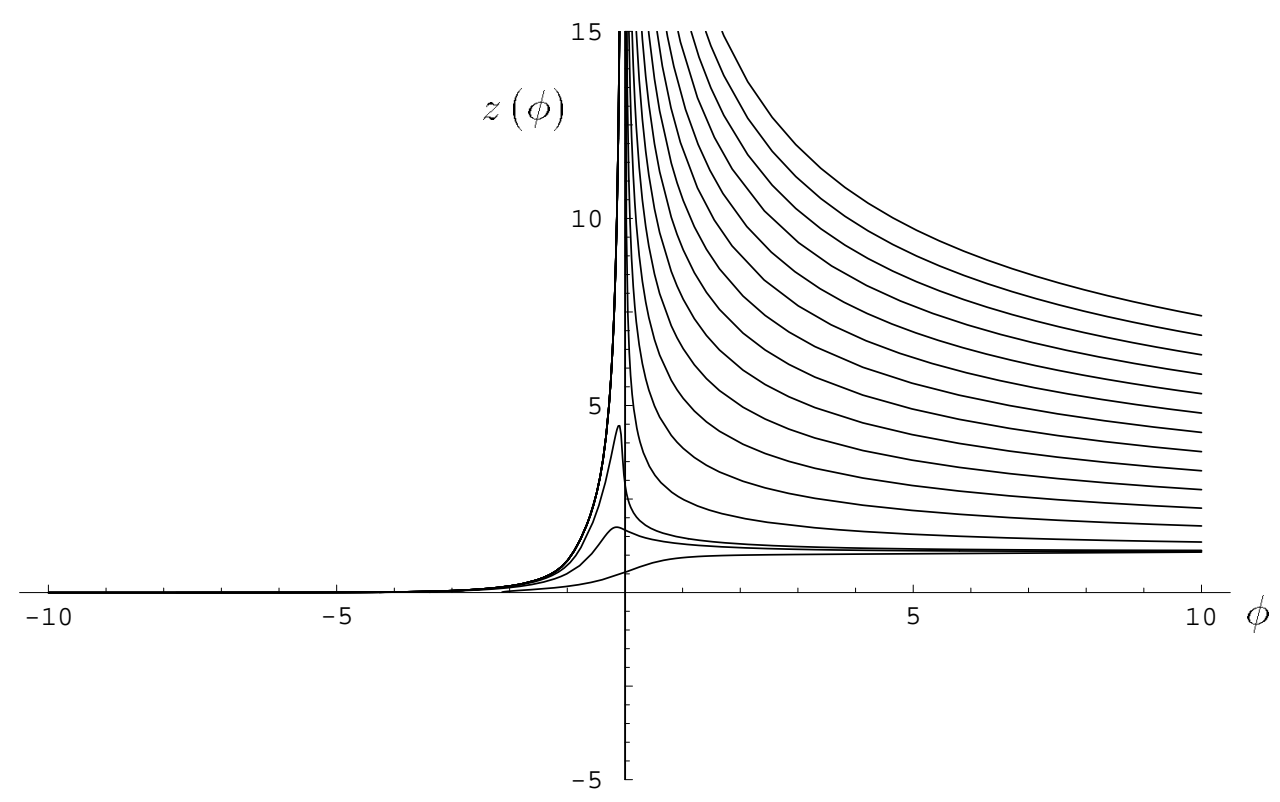

Figure 3: The dependence of the quantity $z$ on the scalar field for a family of non-singular solutions for the case $k=-1, s=-1$ and $\delta=0.5$.

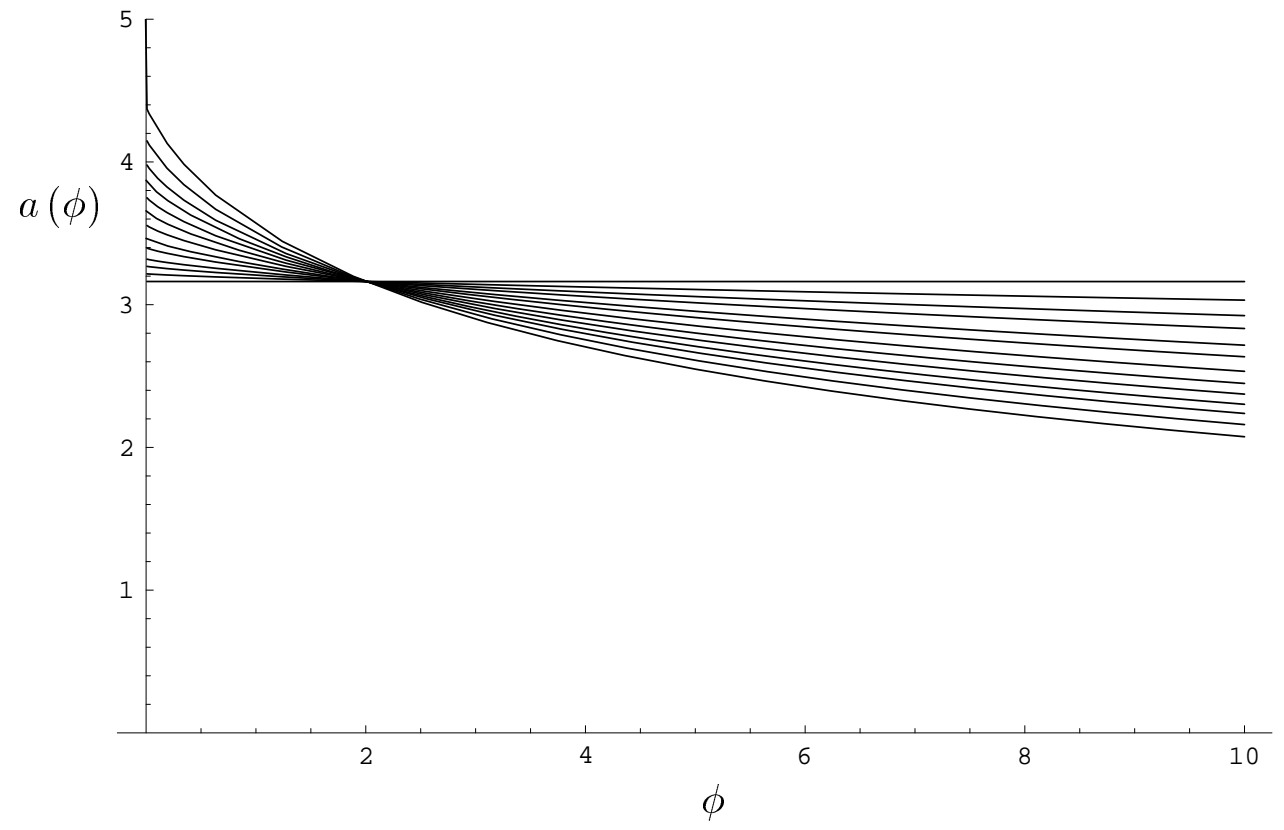

Figure 4: The dependence of the scale factor $a$ on the scalar field for a family of non-singular solutions for the case $k=-1, s=-1$ and $\delta=0.5$. 


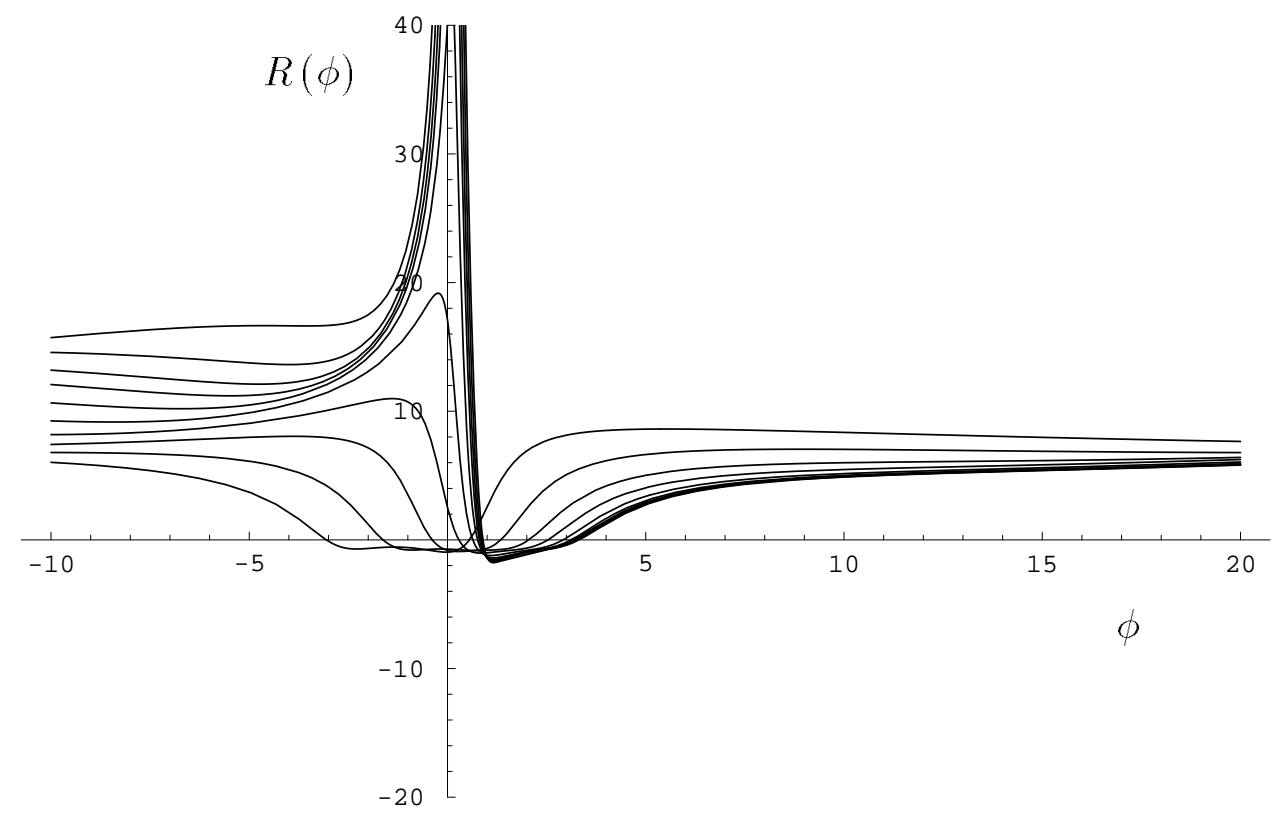

Figure 5: The scalar curvature $R$ versus the scalar field $\phi$ for a family of non-singular solutions for the case $k=+1, s=+1$ and $\delta=0.5$.

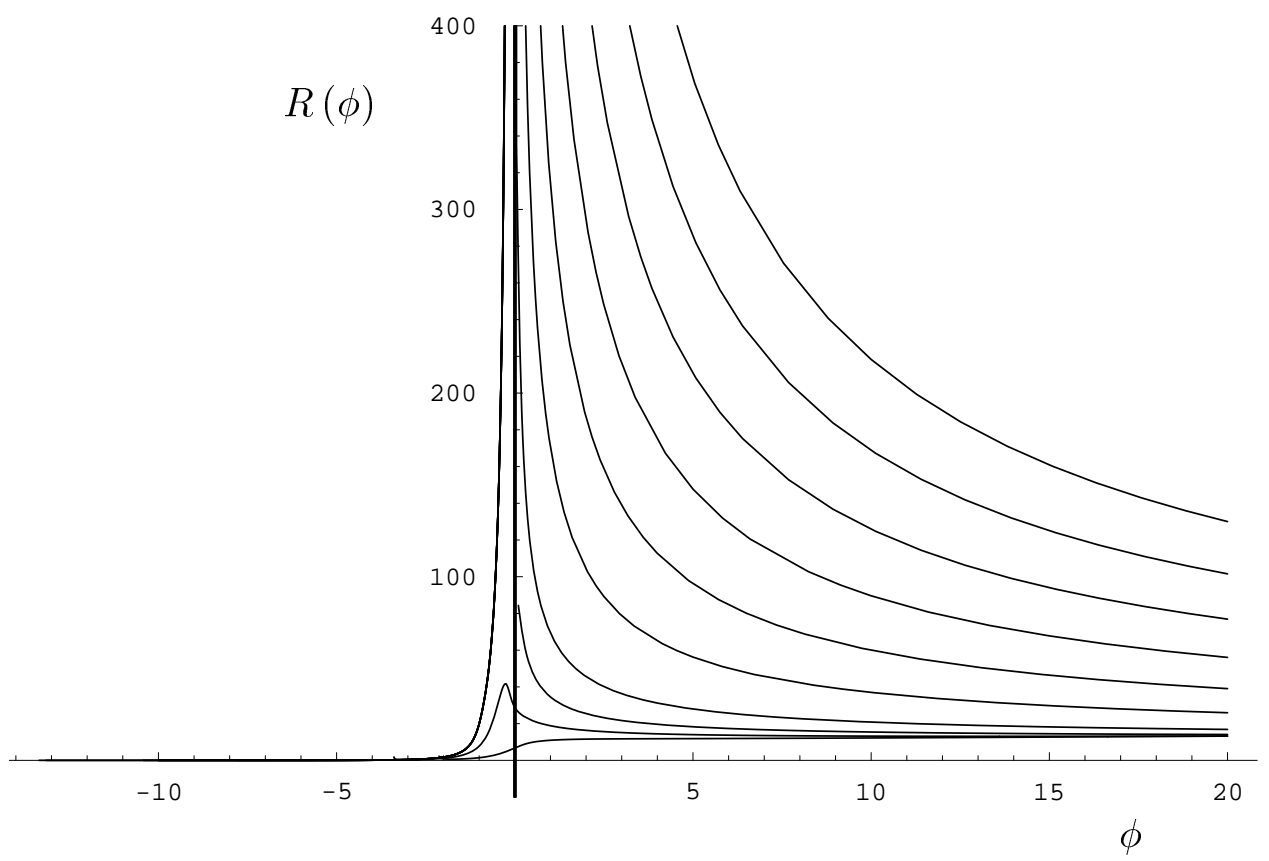

Figure 6: The scalar curvature $R$ versus the scalar field $\phi$ for a family of non-singular solutions for the case $k=-1, s=-1$ and $\delta=0.5$. 